\title{
ABSENCE OF PAIRING MECHANISM DUE TO COUPLING OF OXYGEN HOLES TO LOCALIZED SPINS IN HIGH- $T_{c}$ SUPERCONDUCTORS
}

\author{
Andrzej M. OLEŚ and Jan ZAANEN \\ Max-Planck-Institut für Festkörperforschung, D-7000 Stuttgart 80, Federal Republic of Germany
}

A canonical transformation method is used to derive an effective Hamiltonian which describes $C u(3 d)$ spins and $O(2 p)$ holes within the $\mathrm{CuO}_{2}$ plane in the charge transfer regime. It is found that spin-carrier interactions present in second order overrule the pairing mechanisms as proposed by Emery and Hirsch. The results suggest that mixed valence regime is more realistic for high $T_{c}$ superconductors.

\section{INTRODUCTION}

In the search for an electronic mechanism of high $T_{c}$ superconductivity, a few possibilities have been suggested so far. The most prominent of them is the resonating valence bond (RVB) mechanism of Anderson et al. [1] which assumes that the effective Hubbard Hamiltonian is at work and the doping creates defects in the spin lattice. On the other hand, photoemission data suggest that the holes are created rather in $O(2 p)$ orbitals within $\mathrm{CuO}_{2}$ planes of a high $T_{c}$ superconductor [2]. Two mechanisms of superconductivity based on this picture have been suggested by Emery. [3] and Hirsch [4]. They both should originate from effective carrier-carrier attraction which results from the hybridization $V$ between $C u(3 d)$ and $O(2 p)$ states in fourth order of perturbation theory. Here we investigate whether these mechanisms may indeed be effective in high $T_{c}$ superconductors.

A realistic model Hamiltonian which describes the $C u\left(3 d_{x^{2}-y^{2}}\right)$ and $O\left(2 p_{x(y)}\right)$ orbitals in a high $T_{c}$ superconductor is of the form

$$
\begin{aligned}
& H=\varepsilon_{d} \sum_{i \sigma} n_{d i \sigma}+U \sum_{i} n_{d i \uparrow} n_{d i \downarrow}+\varepsilon_{p} \sum_{j \sigma} n_{j \sigma} \\
& +\sum_{j j^{\prime} \sigma} t_{j j^{\prime}} a_{j \sigma}^{+} a_{j 1 \sigma}+\sum_{<i j>\sigma} V_{i j}\left(d_{i \sigma}^{+} a_{j \sigma}+h . c .\right) .
\end{aligned}
$$

The operators $d_{i \sigma}^{+}$and $a_{i \sigma}^{+}$create holes in a $d_{x^{2}-y^{2}}$ orbital at $C u$ site and in $p_{x(y)}$ orbital at $O$ site, respectively. Kinetic energy is due to the $p-p$ hopping $t_{j j^{\prime}}$ and the $d-p$ hybridization $V_{i j}$. The energy scale is defined by two parameters: (i) the charge transfer energy $\Delta=\varepsilon_{p}-\varepsilon_{d}$ and (ii) the Coulomb integral $U$ for $C u\left(3 d_{x^{2}-y^{2}}\right)$ orbital. Depending on their values, one is in different physical regimes. For large $U$ and $\Delta$, the Hamiltonian (1) either reduces to an effective Hubbard model (if $\Delta \gg U$ ), or a charge transfer correlation gap is obtained (if $U \gg \Delta$ ). If either $U$ or $\Delta$ become of the order of the relevant bandwidth, we enter metallic

* Permanent address: Institute of Physics, Jagellonian University, PL-30-059 Kraków, Poland. regimes, being of either Mott-Hubbard (if $\Delta \gg U$ ) or mixed valence (if $U \gg \Delta$ ) type. The analysis of photoemission data [2] gives evidence that high $T_{c}$ superconducting oxides are characterized in any case by $U>\Delta$. Thus, these systems are either in the charge transfer regime and exhibit a correlation gap, or they are itinerant mixed valence systems. Below we analyze in more detail the physics in the charge transfer regime.

\section{CANONICAL PERTURBATION EXPANSION AND SPIN-CARRIER INTERACTION}

The basic idea of a canonical perturbation expansion is to construct an effective Hamiltonian which replaces interconfigurational hopping processes by effective interactions. In the case of the Hubbard Hamiltonian in the strongly correlated regime it gives an effective RVB Hamiltonian [5]. Here we generalize this method to the Anderson lattice Hamiltonian (1).

In the charge transfer regime, one has localized spins at $C u$ atoms and doping creates holes in $O(2 p)$ orbitals. The zeroth order Hamiltonian $H_{0}$ is just given by the term proportional to the $p-p$ hopping $t=t_{j j^{\prime}}$. There are two transitions which change the configuration of a single $C u$ atom: (i) from a $C u$ site to one of the surrounding $O$ atoms and (ii) from an $O$ atom to $C u$ atom, characterized by the energy difference $\Delta$ and $U-\Delta$, respectively. An effective Hamiltonian may be derived by introducing projection operators which correspond to these configurations [6]. In second order one obtains

$$
\begin{gathered}
H_{e f f}^{(2)}=\left(\frac{1}{\Delta}+\frac{1}{U-\Delta}\right) \sum_{<i j j^{\prime}>} V_{j i} V_{i j^{\prime}} \\
{\left[S_{i}^{z}\left(a_{j \uparrow}^{+} a_{j^{\prime} \uparrow}-a_{j \downarrow}^{+} a_{j^{\prime} \downarrow}\right)+S_{i}^{+} a_{j \downarrow}^{+} a_{j^{\prime} \uparrow}+S_{i}^{-} a_{j \uparrow}^{+} a_{j^{\prime} \downarrow}\right]} \\
+\frac{1}{2}\left(\frac{1}{\Delta}-\frac{1}{U-\Delta}\right) \sum_{<i j j^{\prime}>\sigma} V_{j i} V_{i j^{\prime}} a_{j \sigma}^{+} a_{j^{\prime} \sigma},
\end{gathered}
$$

where $O$ sites $j$ and $j^{\prime}$ are coupled by second order processes. $H_{\text {ff }}^{(2)}$ contains spin-carrier (Kondo-like) interaction and a new $p-p$ hopping term. 
The simplest way to study the consequences of the derived interaction on the electronic states is to use the mean-field approximation. One finds that the quasiparticle energies depend on the underlying magnetic order of the spin lattice. As an example, we have studied a one dimensional chain which consists of interchanging $C u$ and $O$ atoms. In the absence of direct $p-p$ hopping (i.e. at $t=0$ ) and taking $V=V_{i j}$, one finds that the bottom of the band is located at $-2 V^{2} /(U-\Delta)$ and $-4 V^{2} /(U-\Delta)$ in an antiferromagnet and in a ferromagnet, respectively. Thus, one carrier polarizes its surrounding ferromagnetically and leads to the formation of magnetic polaron. The same was observed with finite hopping $t$, as shown in Fig. 1 .

In order to go beyond the mean-field approximation, we have solved exactly a small cluster of two $C u$ and two $O$ atoms on a square, filled with two spins and one $O$ hole. Under neglect of $t$ the quantum fluctuations are quite important. Basing on this observation, Zhang and Rice [7] suggested that the internal degrees of freedom of the $\mathrm{Cu} \mathrm{O}_{4}$ clusters can be neglected and that the system can be mapped on a RVB Hamiltonian. We think that this is not obvious since $t$ is quite large in the real systems and this quantity tends to suppress the fluctuations. Further, one may have doubts concerning the validity of the one step renormalization procedure of Zhang and Rice [7].

\section{DISCUSSION OF FOURTH ORDER PROCESSES}

As pointed out by Emery [3], effective attractive interactions follow from the Hamiltonian (1) in fourth order. Their origin is analogous to that of spin-spin
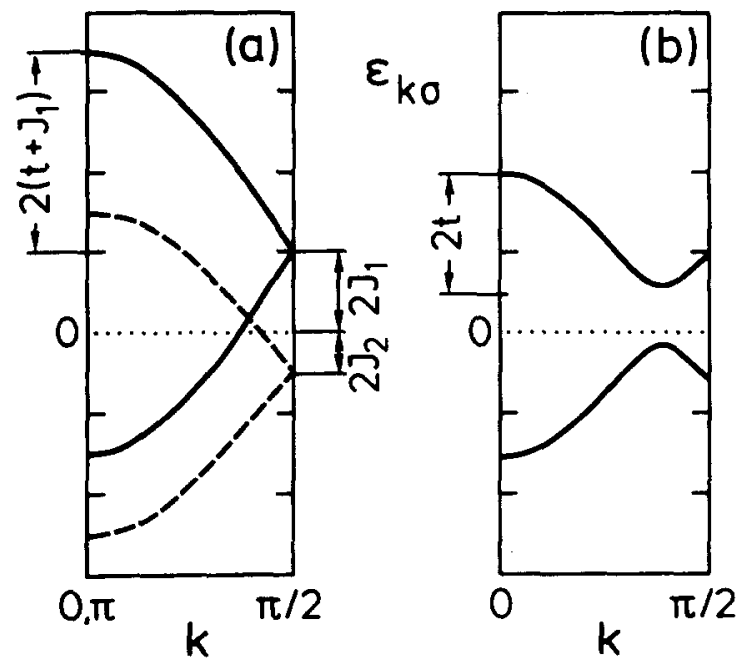

Fig. 1. Quasiparticle energies $\varepsilon_{k \sigma}$ (in arbitrary units) for a one dimensional $C u-O$ chain in mean-field approximation for (a) ferromagnetically and (b) antiferromagnetically ordered $C u$ spins. Solid (dashed) lines in (a) represent majority (minority) spin in the folded Brillouin zone, $J_{1}=V^{2} / \Delta$ and $J_{2}=V^{2} /(U-\Delta)$. superexchange. For instance, in the one dimensional chain of Sec. 2 one finds the effective interaction of the form $-J\left(k, k^{\prime}\right) a_{k^{\prime}}^{+} a_{-k_{1}}^{+} a_{\left.-k^{\prime}\right\rfloor} a_{k^{\prime} \uparrow}$ with $J\left(k, k^{\prime}\right)>0$. Such processes could indeed lead to hole pairing if second order terms would give only band renormalization. In fact, the latter terms give rather complicated dynamics and by simple phase space arguments it can be seen that this results in repulsive interactions of order $V^{2} / \Delta$ and $V^{2} /(U-\Delta)$ and that Emery's interactions are small corrections to those.

In contrast to Emery, Hirsch [4] emphasizes spincarrier interaction. His argument is that a moving carrier creates defects in an ordered spin lattice, while two carrier may hop together without disturbing the magnetic order. There are a couple of reasons that this pairing mechanism is also unlikely. First of all, he assumes that the carrier moves in an antiferromagnetic lattice only due to spin-flip processes, i.e. he considers only transitions of the $1 /(U-\Delta)$ type. In fact, the $1 / \Delta$ processes without spin-flip are of equal importance [8]. Furthermore, the mechanism of pairing based on fourth order processes is again much weaker than the spin-carrier processes which act in the opposite direction. This is also supported by our analysis of spin-spin and spin-carrier interactions in a finite cluster [6].

An important problem is to bridge the gap between the effective RVB Hamiltonian [1] and the two-band model. In view of the presented results, one may have strong doubts that this task is ever possible in the localized (charge transfer) regime. Further, we have shown that one finds also in the two-band model only repulsive interactions on small length scales as in the single band Hamiltonian [9]. The results presented above suggest that two-band model in the localized regime is rather unrealistic for high $T_{c}$ superconductors. In fact, experimental data indicate that a well developed correlation gap is absent. Thus, we think that the charge degrees of freedom are important and one should search for a possibility of electronic mechanism of superconductivity in the mixed valence regime.

\section{ACKNOWLEDGMENTS}

We wish to thank Profs. G.A. Sawatzky and W. Hanke and Drs. O. Gunnarsson and G. Stollhoff for many useful discussions. This work was supported by the Polish research project CPBP 01.09.

\section{REFERENCES}

[1] P.W. Anderson, G. Baskaran, Z. Zou and T. Hsu, Phys. Rev. Lett. 58 (1987) 2790.

[2] Z. Shen et al., Phys. Rev. B 36 (1987) 8414.

3 V.J. Emery, Phys. Rev. Lett. 58 (1987) 2794.

4 J.E. Hirsch, Phys. Rev. Lett. 59 (1987) 228.

5 K.A. Chao, J. Spalek and A.M. Oles, J. Phys. C 10 (1977) L271; Phys. Rev. B 18 (1978) 3453.

[6] J. Zaanen and A.M. Oles, Phys. Rev. B in press (1988).

[7] F.C. Zhang and T.M. Rice, Phys. Rev. B in press (1988).

[8] L.M. Roth, Phys. Rev. Lett. 60 (1988) 379.

[9] G. Stollhoff, Z. Phys. B 69 (1987) 61 . 